The Hewlett-Packard Europhysics Prize for 1979 was presented by Sir Sam Edwards at the Conference on the Physics of Semiconductor Surfaces and Interfaces held in Paris from 26 to 28 November, 1979. Prof. Paige writes here on behalf of all the prize winners namely : Eric A. Ash of London, Jeffrey H. Collins of Edinburgh, Yuri V. Gulaev of Moscow, Kjell A. Ingebrigtsen of Trondheim, Edward G.S. Paige of Oxford.

We are all familiar with surface waves on water and many will have had their first introduction to wave phenomena such as interference and diffraction through observing ripples on the surface of a liquid. The motion characteristic of the wave is a displacement confined to the plane containing the direction of propagation and the normal to the surface (the sagital plane) and, of course, decaying with depth below the surface.

Here we are concerned with the same type of mechanical (acoustic) wave propagating on a crystalline solid. Its displacement is confined to the sagital plane when propagation is along a symmetry direction and, like the wave on a liquid, it combines longitudinal and transverse motion. Its velocity is slightly less than the lowest velocity of a bulk acoustic wave in the medium; it is typically $3 \times 10^{3} \mathrm{~m} \mathrm{~s}^{-1}$, some five orders of magnitude slower than an electromagnetic wave. In the frequency range of interest $(<2 \mathrm{GHz})$ dispersion is negligible. Below the surface, the wave motion decays with a characteristic length approximately equal to a wavelength. Thus at $10 \mathrm{MHz}, 100 \mathrm{MHz}$ and $1 \mathrm{GHz}$ the wavelength (decay length) is $300 \mu \mathrm{m}, 30 \mu \mathrm{m}$ and $3 \mu \mathrm{m}$, respectively. Provided the crystal surface receives a good "optical" polish, scattering due to surface irregularities can be neglected. Absorption of the wave increases approximately as the square of the frequency and in commonly used materials is negligible except at the highest frequencies of interest $(>1 \mathrm{GHz})$ and for long delay paths $(>30 \mathrm{~cm})$.

\title{
Surface Acoustic Waves and Their Applications
}

\author{
E.G.S. Paige, Oxford \\ (Dept. of Engineering Science)
}

Surface acoustic waves (SAW) on solids were placed on a firm physical footing by Rayleigh ') who, in 1885, predicted the dispersive relation and other characteristics of a wave propagating on a plane surface of an isotropic elastic solid. For the next eighty years, interest in these waves was sustained by geophysicists and a few acousticians. Then, in the midsixties, an explosion of interest began when it was realized, and initial feasibility experiments confirmed, that a solid supporting surface acoustic waves could be used to process signals in electronic systems - the SAW signal processing device came into being. Now SAW devices operating in the $20 \mathrm{MHz}$ to $2 \mathrm{GHz}$ frequency range are incorporated in many electronic systems, ranging from TV and radar to electronic warfare systems. It is the exceptionally large bandwidth (up to about $1 / 2 \mathrm{GHz}$ ) allied with low attenuation (high Q) which gives the SAW device its attractive performance. In addition, compared with its competitors, the SAW device is relatively easy to fabricate.

As background, we begin by describing a simple device structure.

\section{SAW Devices}

The surface acoustic wave devices which have been produced perform their function within an electronic system, a television or radar set, for example. It is therefore necessary to convert an electrical signal to an acoustic wave and then to reconvert it to an electrical signal. The type of transducer usually employed to perform the conversion is the interdigital

\section{No Decision Yet on the Communities' Fusion Programme}

Decisions on the funding of fusion research in the Communities over the five years 1979-1983 were in principale to have been taken at the meeting of the Council of Ministers - Research on 2 October. Unfortunately, not for the first time one programme has been held up because of a failure to reach agreement on another (notably the Joint Research Centre programme) but it is hoped that the block can be cleared on 20 December. The current 5-year plan runs from 1976-1980 so there is no danger of an immediate hiatus. Nevertheless, the problem of ensuring continuity is underlined. The figure discussed is for an expenditure of around 700 M EUA, not including JET, for which a price indexing formula has been agreed by the JET Council that is unlikely to be called into question. The balance to be struck between physics and technology extent to which co-operation on laser compression, can be included. has caused some difficulty as well as the

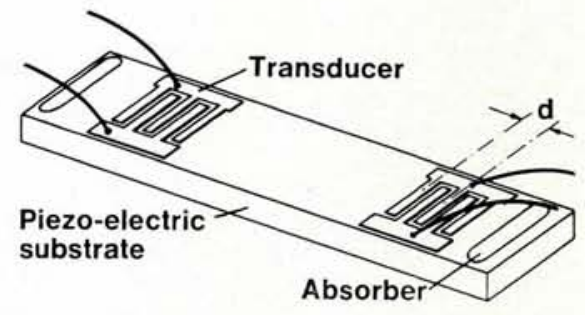

Fig. - Schematic diagram of a surface acoustic wave delay line. (d) is the periodic length in the transducer.

transducer, an example of which is shown in Fig. 1. It is formed by photolithography in a thin $(\sim 1000 \AA)$ metal film, aluminium for example, deposited on a piezoelectric crystal. When a voltage is applied between the busbars of the transducer, the crystal between and under the fingers becomes strained. If the voltage alternates, the strain alternates and each pair of fingers becomes the source of a wave. As is evident from the figure, the sources are stacked one behind the other and interference between the radiated waves occurs. This interference determines the frequency response of the transducer. For the periodic structure shown, peak response occurs at a frequency at which the surface wave wavelength is equal to the periodic length of the transducer. The frequency response may be varied by choice of relative position of the fingers and/or by the extent of their overlap. Because the launched wave on the piezo-electric substrate is accompanied by electric fields, a second interdigital transducer may detect its presence. The combined two-transducer structure shown in Fig. 1 acts, therefore, as a band-pass frequency filter. Currently, one of the most important SAW devices is such a filter for the IF stage of colour TV receivers. Fig. 2 shows a typical TV filter and its frequency response. Production of these devices in Europe and elsewhere in the world runs into several million units annually.

For other applications delay lines are required and the low propagation velocity of the surface acoustic wave enables very compact delay lines to be fabricated. The delay time is determined by separation of the trans- 

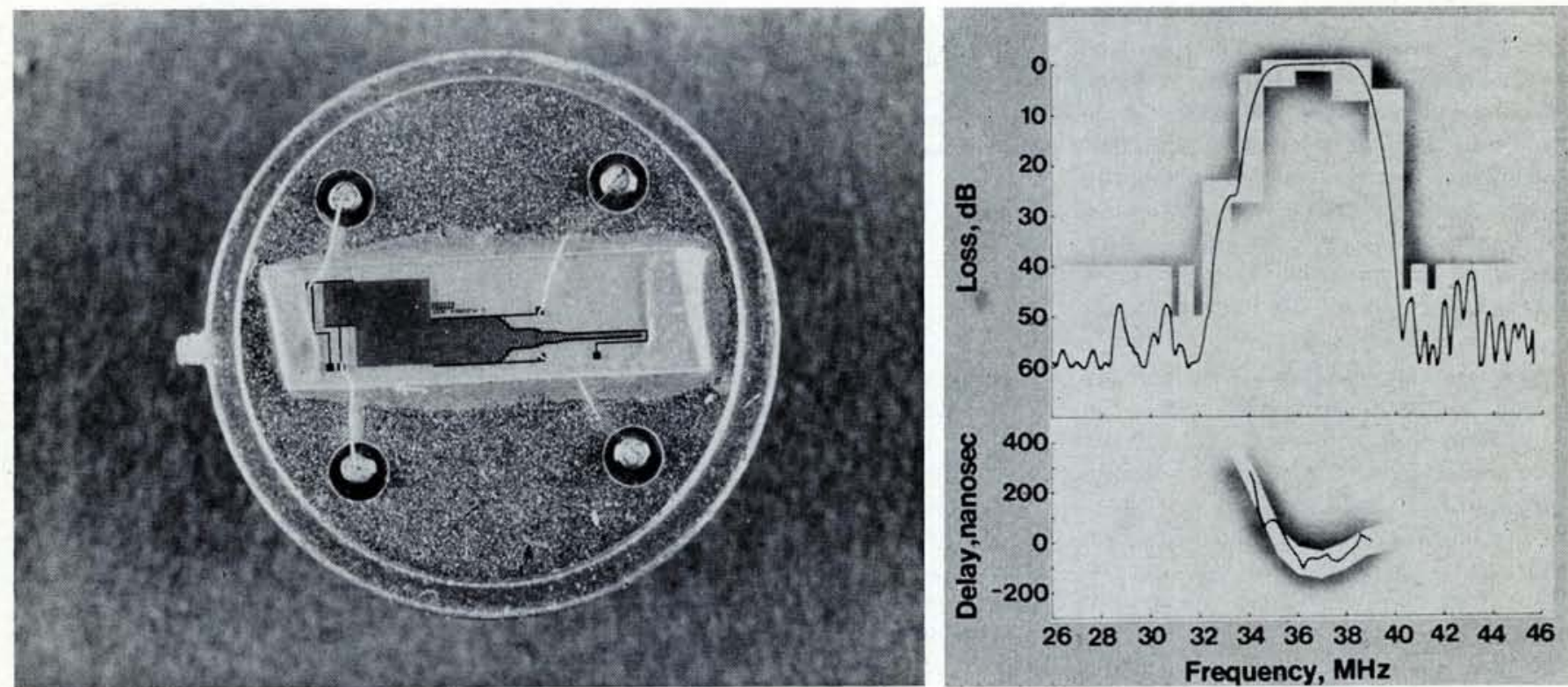

ducers, and approximately $3 \mu \mathrm{s}$ of delay is produced for every centimetre of separation. Because the wave propagates on the crystal surface, several transducers may be placed in the path of a launched wave producing a tapped delay line.

The examples given above show the transducer performing two roles transducting between electrical and acoustic energy and determining the response of the device. We shall see that structures other than transducers may affect the overall behaviour of the SAW device. For example, if a periodic set of shallow, parallel grooves is made in the surface, then they may act as a frequency dependent mirror to an incident surface wave. (The Bragg condition gives the frequency of peak reflection). Placed between input and output transducers such a structure can be used to determine frequency response.

Interesting interactions may be obtained between the surface wave and free electrons such as may be present in a semiconductor in close proximity to the piezo-electric surface. The electric field accompanying a surface wave on a piezo-electric surface, produces periodic variation in electrical potential in the vicinity of the surface. Free electrons tend to seek the potential minima and in doing so get dragged along by the propagating wave - they receive a net acceleration force from the wave. This particular acousto-electric effect leads to attenuation of the acoustic wave. Acoustic energy is transferred from the surface wave to free electrons which in turn lose it in random collision events with the lattice. However, if an applied voltage produces an electric field which causes the electrons to drift with supersonic velocity, then in the presence of the wave they receive a net decellerative force and energy is transferred from electrons to the wave. In this way a surface acoustic wave amplifier can be made. Other related acoustoelectric phenomena will be discussed later in this article.

We now turn to consider some specific aspects of surface waves. The reader wishing to gain a wider background to surface waves and devices should consult the general references $2,3,4,5$ ).

\section{Propagation \\ of Surface Acoustic Waves}

All crystalline solids are acoustically anisotropic. As a consequence propagation properties differ significantly not only from one material to another but from one cut and propagation direction to another. On commonly used cuts of quartz, for example, diffraction occurs more rapidly than on an isotropic substrate while on lithium niobate it occurs less rapidly $\left.{ }^{6}\right)$. Off symmetry directions, beam steering (non-parallelism of propagation vector and poynting vector) can be dramatic. Clearly, in transducer design and layout a knowledge of the basic propagation properties of the substrate material is essential.

Recently it has been found that a piezo-electric solid will support not only the previously described Rayleigh mode but also a surface wave in which the motion is entirely transverse and in the plane of the surface. The new mode is known as the BleusteinGulaev wave after its independent discoverers ${ }^{7}$ ). Unlike the Rayleigh mode it penetrates deep into the solid but a thin metal overlay on the insulating substrate reduces the penetration to one or two wavelengths.
Fig. 2 - Surface wave filter for TV manufactured by the Plessey Company, U.K. Left - View of the device. Right - Performance of the device (curves) falling in the specification "window".

Choice of which mode is launched can be made by crystal cut, propagation direction and frequency. Unless otherwise stated reference to a surface wave in this article will imply the Rayleigh mode.

\section{Generation and Detection of Surface Waves}

While there are a variety of ways of launching and sensing surface acoustic waves, the interdigital transducer of Fig. 1 is used almost exclusively. It was the demonstration ${ }^{8}$ ) in 1969 that surface acoustic waves could be launched and detected with the interdigital transducer with near $100 \%$ conversion efficiency and fractional bandwidths of $20 \%$ or more which lit the fuse to the explosion of activity on SAW devices.

As can be seen from Fig. 1, when the interdigital transducer is placed on an insulator it will behave electrically like a capacitor. If the insulator is piezo-electric, then in general, acoustic waves can be launched as described in the introduction and the transducer will exhibit a radiation resistance in common with any radiating aerial. Electrically, the transducer has a combined reactive and resistive component. The reactive component can be tuned out by an inductor and the resistive component matched to the source and driving line impedance. (Matching is achieved by choice of aperture, e.g. 100 wavelength aperture on lithium niobate produces a $50 \Omega$ radiation resistance). Under the tuned and matched conditions, $100 \%$ conversion efficiency 
can occur. The important question of the fractional bandwidth over which it can be maintained is a strong function of the appropriate elements of the piezo-electric tensor which depend on crystal cut, propagation direction as well as choice of material. For $100 \%$ conversion efficiency, fractional bandwidths of $25 \%$ on lithium niobate and $5 \%$ on quartz ${ }^{8}$ ) are achievable. Through transducer design, the bandwidth can be increased if conversion efficiency is sacrificed.

The simple SAW structure in Fig. 1 can be made using lithium niobate with the following performance: fractional bandwidth $20 \%(N=5)$; insertion loss $7 \mathrm{~dB}$; centre frequency $100 \mathrm{MHz}$; delay time 10!s. Of the insertion loss, $6 \mathrm{~dB}$ is due to the bidirectionality of the transducer - from symmetry only half the acoustic power is launched in the direction of the receiving transducer - the remaining $1 \mathrm{~dB}$ arises partly from diffraction and partly from circuit losses.

Various physical phenomena complicate the performance of the interdigital transducer. For example, the metal fingers can reflect the surface wave by the effective impedance change they create due to mechanical, electrical and topographical changes at the surface. Regeneration of surface waves by a wave launched from one part of the transducer being sensed by another part as it propagates under it is a further complication ${ }^{9}$ ). However, by choice of material and elaboration of the transducer design, the problems can be reduced to negligible proportions in many cases, but as the demand for yet improved performance continues, the pressure for a more complete theoretical model of transducer behaviour grows.

\section{Surface Structures}

The dual role placed on the interdigital transducer in a device, mentioned in the introduction, may be made less demanding by placing surface structures in the path of the acoustic beam.

Fig. 3 shows examples of various surface structures which may be formed by grooves or by deposition of metal or insulating films. For their performance the structures in Figs. $3 a$ and $3 \mathrm{~b}$ depend on satisfying the Bragg reflection condition at a given frequency. In Fig. 3a the reflectors are equally spaced and when the frequency is such that the acoustic wavelength equals half the periodic repeat distance in the array, very strong reflection can occur. The structure forms a resonator cavity ${ }^{10}$ ) and re-

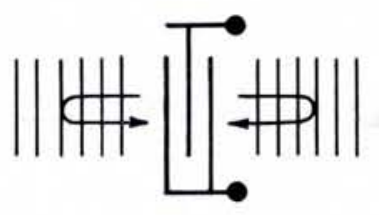

(a)
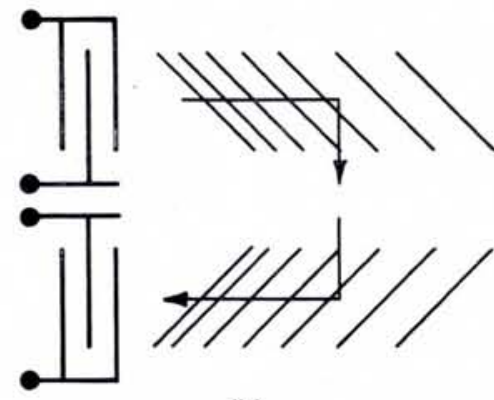

(b)

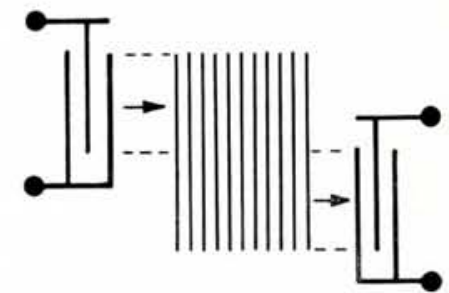

(c)

Fig. 3. - Surface structure: (a) Reflectors for a resonator; (b) reflectors for a dispersive delay line; (c) multistrip coupler; (d) surface waveguide.

presents a means of utilizing the very low attenuation of the substrate material to achieve very narrow frequency band filters. The structure shown in Fig. $3 b$ is not only different in that each array is designed to reflect through $90^{\circ}$ but also, in one of its most important applications, namely dispersive delay lines, the separation between reflectors is progressively changed. In the example shown, high frequency waves get reflected round a shorter path than low frequency waves. The dispersion can be made a tailored function of frequency ${ }^{11}$ ) by choice of spacing between reflectors. We shall refer to an important application of dispersive delay lines later.

The surface structure shown in Fig. $3 \mathrm{c}$ is known as the multistrip coupler (MSC). Made with strips of metal on a piezo-electric substrate, it performs as a directional coupler. Waves launched from one transducer illuminate half the coupler and create potential differences between strips which, in turn, generate an acoustic wave in the originally unilluminated half of the coupler ${ }^{12}$ ). Up to $100 \%$ of the incident wave energy can be transferred, the actual fraction depending on the number of strips, their spacing, the wavelength of the incident wave and the electromechanical properties of the material. On a favourable crystal cut of lithium niobate $100 \%$ transfer can be achieved with 90 strips. While many applications of this structure have been proposed ${ }^{12}$ ), it finds most widespread use in suppressing spurious modes in band-pass frequency filters. It is incorporated in the TV filter shown in Fig. 2, for example.
The accessibility of the surface wave lends it to further manipulation. For instance, a thin film deposited on the surface of the substrate may raise or lower the surface wave velocity. If the velocity is lowered then, with the aid of photolithography, a waveguide structure can be formed such as the simple example shown in Fig. 3 d. The concept of guiding a surface acoustic wave has been amply demonstrated and various analogues of electromagnetic microwave components have been constructed.

\section{Surface Wave Acousto-electric Effects}

Following work early in the 1960s on the amplification of bulk waves, it was natural that the possibility of amplification of surface acoustic waves be explored. An early, impressive, demonstration ${ }^{14}$ ) came in 1968 in which a surface wave propagating on lithium niobate was amplified by electrons in a drift field in an adjacent slab of silicon. An air gap of less than $1 \mu \mathrm{m}$ was left between the silicon and the lithium niobate to avoid mechanical damping of the wave. Terminal gain of $7 \mathrm{~dB}$ was observed when electrons had a drift velocity about twice the surface wave velocity.

The acousto-electric interaction leading to amplification has already been mentioned. Amplification of a surface wave as described above is complicated because two materials are involved and because of the twodimensionality of the geometry. In a detailed analysis ${ }^{15}$ ) it is found that superimposed on the drift motion of the electrons is a circulating loop motion accompanied by an "acoustoelectric" voltage transverse to the 

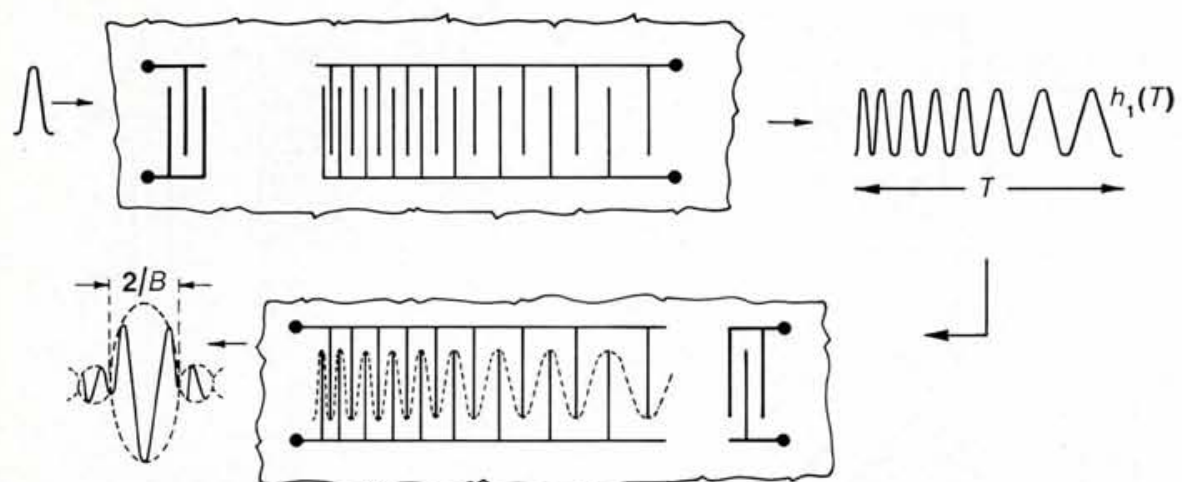

Fig. 4. - Dispersive delay lines and pulse compression. Impulsing the upper delay line produces a frequency modulated pulse $h_{1}(t)$. Fed into the lower delay line it produces an auto-correlation peak.

drift voltage. A close parallel may be drawn with surface waves on water amplified by the wind.

While various other amplifying structures have been studied, more recently interest has turned to acoustoelectric effects involving semiconductor junctions and the possibility of signal storage in them. An early example of this is the "Schottky Diode Memory Correlator" $\left.{ }^{16}\right)$. The device had essentially the same geometry as the amplifier except that the surface of the silicon nearest to the lithium niobate substrate was covered with a matrix of diodes. The diodes could be biased by a common pair of electrodes which sandwiched the silicon/ air gap/niobate structure. By applying a very short duration forward bias, effectively a "snap shot" was taken of the field distribution (due to a surface wave) under the array, the information being stored as charge on the diodes. If at some later time another surface wave was launched under the array, the non-linearity of the diode characteristic resulted in a multiplication of locally stored and passing signals. The output at the common pair of electrodes was an integration of the local multiplication products and consequently gave the cross-correlation between the stored and passing signal. Thus in the one device, signal storage and crosscorrelation between a pair of signals can be performed.

\section{Devices and Their Applications}

The interest of virtually all the major electronics companies in the world concerned with advanced systems has been caught, during the past decade, by the realization that reproducible, reliable SAW devices can be made with exceptional performance. The consequent intense effori has resulted in a rich variety of device structures and types. One general feature which has emerged is the suitability of SAW devices for radar - the parameters fit well with present requirements and those of the near future. In normal communication systems, signal lengths are long, bandwidths are narrow and flexibility is desirable - these requirements are better met by conventional and other technologies (digital IC, charge coupled devices).

Two of the most successful surface wave devices to emerge are the bandpass filter for colour TV and dispersive delay lines for radar pulse compression. The first has been discussed in the introduction. Here we shall briefly describe the second and its application.

In the top half of Fig. 4, a simple form of dispersive delay line is displayed together with its impulse response $h_{1}(t)$. If this is fed into a second dispersive delay line, identical to the first except that its impulse response is the time reverse of $h_{1}(t)$, then at one particular time the position of the wave is such (shown in the sketch) that a pronounced output peak occurs. This is in fact the autocorrelation peak of $h_{1}(\mathrm{t})$. A frequently used form of $h_{1}(\mathrm{t})$ is :

$$
\begin{gathered}
h_{1}(t)=A \cos \left(\omega t+b t^{2}+c\right) \\
\text { for } t_{1} \leq t \leq t_{1}+T ; \\
\text { otherwise } 0
\end{gathered}
$$

This has an instantaneous frequency $(\delta \Phi / \delta t)$ bandwidth $B$ of $2 b T$. The inverse of this is the width of the auto-correlation peak. Simple energy arguments show that increase of peak signal level relative to noise (which is uncompressed) is $B \times T$. For a radar which sends out a simple pulse $(b=0)$ to achieve the same performance (signal-to-noise, width of pulse) the power at the transmitter would have to be raised by $B T$. However, because a radar is usually peak power limited, we only have to assume the radar was already operating at its peak power limit to make it obvious that the $B T$ increase is not technically pos- sible. Put round the other way, the technique, called pulse compression, can extend the range of a radar without sacrificing other performance factors.

Surface wave pulse compression filters have been made to various designs ${ }^{2,3}$ ), two of which have been sketched in Figs. 3b and 4. Near ideal performance has been achieved with $B T$ products ranging from 10 to $10^{4}$. As a consequence, almost without exception, new pulse compression radars have incorporated surface wave devices.

\section{Conclusion}

In little more than the past decade surface acoustic waves on crystalline solids have passed from one of the more placid back waters of physics (hardly a ripple showed !) to the forefront of modern signal processing technology. It has been - and still is - a good example of "goal-oriented" physics; the results have not been achieved without extensive application of basic physical principles and a detailed knowledge of the physical processes involved.

Finally, in this article I have brought together a (biased) selection of topics relating to surface waves. It has been necessary to omit important developments and references to many significant contributions. The reader is referred to the previously cited ${ }^{3,4,5}$ ) reviews for a more complete coverage of the topic.

\section{REFERENCES}

1. Rayleigh Proc. London Math. Soc. 17 (1885) 4.

2. White, R.M. Proc. IEEE, 58 (1970) 1238.

3. Maines, J.D. and Paige, E.G.S. IEE Rev. 120 (1973) 1078.

4. Maines, J.D. and Paige, E.G.S. Proc. IEEE 64 (1976) 639.

5. Oliver, A.A. edit. Topics in Applied Physics 24 (Springer-Verlag) 1978.

6. Mason, I.M. and Ash, E.A. J.Appl.Phys. 42 (1971) 5343.

7. Gulaev, Y.V. Sov.Phys. - JETP Letts. 9 (1969) 63.

8. Smith, W.R., Gerard, H.M., Collins, J. H., Reeder, T.M. and Shaw, J.H. IEEE Trans. MTT-17 (1969) 856.

9. Ingebrigtsen, K.A. Proc. Ultrasonic Symp. (1972) 403

10. Ash., E.A. IEEE Symposium on Microwave Theory and Techniques, Newpor Beach, Calif., 1970.

11. Williamson, R.C. Proc. IEEE 64 (1976) 702.

12. Marshall, F.G., Newton, C.O. and Paige, E.G.S. IEEE MTT-21 (1973) 206 and 216

13. Ash, E.A. and Morgan, D.P. Electronics Letts. 3 (1967) 462.

14. Collins, J.H., Lakin, K.M., Quate, C.F. and Shaw, J.H. Appl.Phys.Letts. 13 (1968) 314

15. Gulaev, Y.V., Karabapov, A.Y., Kmifa, A.M., Medved, A.V. and Toursunov, S.S. Sov.Phys. Solid State 12 (1970) 2595.

16. Ingebrigtsen, K.A. Proc. IEEE 64 (1976) 764 